\title{
Globe
}

Revue internationale d'études québécoises

\section{Robert Mager et Serge Cantin (dir.), Modernité et religion au Québec. Où en sommes-nous?, Québec, Les Presses de l’Université Laval, 2010}

\section{David Koussens}

Volume 13, numéro 2, 2010

URI : https://id.erudit.org/iderudit/1001139ar

DOI : https://doi.org/10.7202/1001139ar

Aller au sommaire du numéro

Éditeur(s)

Globe, Revue internationale d'études québécoises

ISSN

1481-5869 (imprimé)

1923-8231 (numérique)

Découvrir la revue

Citer ce compte rendu

Koussens, D. (2010). Compte rendu de [Robert Mager et Serge Cantin (dir.), Modernité et religion au Québec. Où en sommes-nous ?, Québec, Les Presses de l’Université Laval, 2010]. Globe, 13(2), 202-204.

https://doi.org/10.7202/1001139ar d'utilisation que vous pouvez consulter en ligne.

https://apropos.erudit.org/fr/usagers/politique-dutilisation/ 


\section{Robert Mager et Serge Cantin (dir.) \\ Modernité et religion au Québec. Où en sommes-nous?, Québec, Les Presses de l'Université Laval, 2010.}

Dirigé par Robert Mager et Serge Cantin, cet ouvrage collectif correspond aux actes d'un colloque organisé en 2008 à l'Université Laval dans le cadre des travaux de l'équipe de recherche Modernité et religion au Québec. Modernité - Religion - Québec: il s'agit là de trois termes ou trois problématiques qui renvoient également aux trois inconnues d'une équation balisant les réflexions et analyses proposées dans l'ouvrage. Tout au long de celui-ci, cette équation, qui semble a priori improbable, s'avère au contraire pertinente pour mettre en lumière les zones d'ombres, les tensions ou l'ambiguïté des relations que la société québécoise entretient avec le phénomène religieux.

Cet ouvrage s'ancre en effet dans la conviction que la religion est un révélateur de la société québécoise et de son devenir. Il ne tente donc pas de dresser un portrait global du phénomène religieux dans le Québec moderne, il propose plutôt des clés pour comprendre la société québécoise au prisme des spécificités historique, culturelle, identitaire, etc. de son rapport au religieux. L'objectif est atteint dans une large mesure, et cela pour deux raisons principales. D'abord, parce que l'ouvrage rassemble les contributions d'une trentaine de chercheurs issus des disciplines les plus variées (histoire, sciences religieuses, droit, philosophie, littérature, théologie ou sociologie) et questionne ainsi la société québécoise au regard d'un prisme commun (le religieux) mais à partir d'approches théoriques et méthodologiques fort diversifiées. Ensuite, parce que malgré leur hétérogénéité, ces contributions s'organisent d'une manière cohérente dans cet ouvrage dont la disposition favorise la compréhension d'un champ d'étude d'autant plus sensible qu'il est souvent l'objet de controverses dans l'actualité. C'est donc désormais principalement sur l'économie générale de ce volume que nous proposons de revenir. En effet, si le format du présent texte ne permet pas d'aborder précisément chacun des vingt-quatre chapitres de l'ouvrage, il autorise néanmoins d'en mettre en perspective l'ordonnancement général. Et l'on indiquera principalement que, sur ce plan, le livre dirigé par Robert Mager et Serge Cantin suit essentiellement une séquence temporelle.

L'ouvrage se penche en premier lieu sur le terreau dans lequel se sont enracinées les relations entre religion et société québécoise au cours du 
$\mathrm{XX}^{\mathrm{e}}$ siècle. Dans cette perspective, la première partie est consacrée à la "sortie de la religion" au cours de la Révolution tranquille, et plus précisément aux processus de sécularisation, à la fois interne et externe, qui peuvent être décelés pendant cette période. Ce tableau historique dressé par les premières contributions de l'ouvrage amène à la deuxième partie du volume qui traite des métamorphoses du religieux dans la période contemporaine.

Cette partie s'ouvre par une contribution d'importance, signée par E-Martin Meunier, J-F. Daniel et J-C. Demers, qui analyse en profondeur les recompositions du catholicisme culturel québécois de la Révolution tranquille à nos jours. Consacrée à la période contemporaine, cette section de l'ouvrage explore en outre les transformations du paysage religieux aussi bien à partir d'études empiriques (D. Cohen-Reis, A. Kenedy et F. Parent) que théoriques (L. Rousseau et J-P. Doucet). Elle se penche également sur les représentations sociales qui découlent de ces mutations du religieux, qu'il s'agisse des représentations que les Québécois se font du fait religieux ou de celles qu'ils se font d'eux-mêmes en fonction de leur rapport au religieux. Cette deuxième partie dresse ainsi un portait de la réalité religieuse qui, sans s'avérer exhaustif - une telle démarche étant probablement vaine dans ce domaine -, est néanmoins éclairant par la diversité des perspectives présentées.

Les troisième et quatrième parties de l'ouvrage ne portent plus sur le processus de sécularisation de la société québécoise, mais bien sur celui de laïcisation de l'État et des institutions publiques depuis les années 1970. Elles s'ancrent donc véritablement dans les débats et controverses actuels relatifs à la place du religieux dans la sphère publique. Et c'est d'ailleurs probablement pour cette raison que la section intitulée «La religion et l'État» (troisième partie) propose au lecteur des contributions de factures inégales. On pourra ainsi regretter que certaines d'entre elles semblent procéder davantage du débat d'idées qu'elles n'adoptent une vraie posture scientifique d'analyse de la réalité juridique des aménagements de la laïcité au Québec. On soulignera toutefois l'apport des travaux de C. Landheer-Cieslak qui retrace les dynamiques parallèles d'autonomisation du droit civil à l'égard de la religion mais également de reconnaissance des religions par ce même droit. Mentionnons aussi l'intérêt de la contribution théorique de D. Jeffrey sur le principe de laïcité au regard de la notion de tolérance.

La quatrième partie se penche sur une institution particulière, l'école publique, dont elle aborde le processus de lä̈cisation sous plusieurs angles. Y sont notamment inclus les chapitres commis par J. Racine qui analysent le processus de mise en œuvre du cours d'éthique et culture religieuse et par J. Cherblanc, lequel explore les transformations du religieux 
et de la place qui lui est conférée dans le système scolaire depuis les trente dernières années.

L'ouvrage se clôt par une cinquième partie, plus prospective, constituée d'une série d'articles qui ouvrent la réflexion et projettent le débat dans l'avenir, cela à partir de réflexions pourtant guidées par la lecture d'auteurs que l'on pourrait déjà qualifier de "classiques» en sciences sociales.

Au final, on pourra dire que ce volume n'est pas une simple contribution de plus sur le phénomène religieux en contexte québécois. A l'inverse, si la perspective pluridisciplinaire de l'ouvrage ouvre l'horizon du lecteur, on soulignera également l'intérêt de réunir dans un même volume les contributions d'auteurs - jeunes chercheurs ou auteurs dont l'expertise est déjà plus reconnue - défendant des positions parfois antagonistes dans le débat social. Il ne s'agit donc pas d'un livre qui clôt la réflexion, mais bel et bien d'un livre qui élargit les perspectives en proposant des outils divers et variés au lecteur soucieux de comprendre les transformations de la société québécoise sans en omettre l'un des principaux facteurs: le rapport des Québécois au religieux.

David Koussens

European University Institute (Italie)

\section{Paul Beaucage \\ Gilles Groulx, le cinéaste résistant, Lux Éditeur, Montréal, 2009.}

Un livre ordinaire sur un cinéaste exceptionnel. Gilles Groulx mérite certainement mieux. On lui avait déjà consacré des études, que l'auteur ne discute ni ne cite, pas plus qu'il ne cite les importants ouvrages sur le cinéma québécois qui traitent de Groulx et son œuvre. Au final il reste un livre un peu bancal; invitation à faire mieux, ce ne sera pas difficile.

L'ouvrage de Beaucage est divisé en quinze chapitres, suivant les quinze films réalisés par Groulx. Ces quinze films sont répartis en trois parties: les années d'apprentissage, l'émergence d'un grand cinéaste, liberté et restriction de l'engagement politique. Cette périodisation est discutable, mais passons pour l'instant. Le tout est amorcé par une trop brève introduc- 